\title{
Evaluating the Impact of COVID-19 Pandemic on The Physicians' Psychological Health; A Scoping Systematic Review
}

\section{Shaista Salman Guraya}

Royal College of Surgeons in Ireland Medical University of Bahrain

\section{Prianna Menezes}

Royal College of Surgeons in Ireland and Medical University of Bahrain

Isabell Nelson Lawrence

Royal College of Surgeons in Ireland and Medical University of Bahrain

Salman Yousuf Guraya ( $\square$ salmanguraya@gmail.com )

University of Sharjah College of Medicine https://orcid.org/0000-0001-5183-023X

Fiza Rashid-Doubell

Royal College of Surgeons in Ireland and Medical University of Bahrain

\section{Research Article}

Keywords: Covid-19, physician, psychological health, personal wellbeing, professionalism

Posted Date: March 7th, 2022

DOI: https://doi.org/10.21203/rs.3.rs-1010519/v1

License: (9) This work is licensed under a Creative Commons Attribution 4.0 International License. Read Full License 


\section{Abstract}

\section{Background}

COVID-19 has endangered healthcare systems at multiple levels worldwide. Published data suggests that moral dilemmas faced during these unprecedented times have placed physicians at the intersections of ethical and unethical considerations. This phenomenon has questioned the physicians' morality and how that has affected their conduct. Our review taps into the spectrum of the transforming optics of patient care during the pandemic and its impact on psychological wellbeing.

\section{Methods}

We adopted the Arksey and O'Malley's framework, defining research questions, identifying relevant studies, selecting the studies using agreed inclusion and exclusion criteria, charting the data, and summarizing and reporting results. Databases of PubMed/Medline, Web of Science, Scopus, Science Direct, CINAHL, and Psyclnfo were searched using a predefined search string. The retrieved titles and abstracts were reviewed. Later, a detailed full-text analysis of the studies which matched our inclusion criteria was performed.

\section{Results}

Our first search identified 875 titles and abstracts. After excluding duplicates, irrelevant, and incomplete titles, we selected 28 studies for further analysis. The total sample size in 28 studies was 15,509 with an average sample size of 637 per study. Both quantitative and qualitative approaches were used, with cross-sectional surveys being utilized in all 16 quantitative studies and the most common qualitative study design used semi-structured interviews. A number of discrete codes were generated which led to the identification of five main themes; mental health, individual challenges, decision-making, change in patient care, and support services.

\section{Conclusion}

This scoping review reports an alarming rise in psychological distress, moral injury, cynicism, uncertainty, burnout and grief among physicians during the pandemic. Decision-making and patient care was mostly regulated by rationing, triaging, age, gender and life expectancy. Poor professional controls and institutional services potentially led to physicians' crumbling wellbeing. This research calls for the remediation of the deteriorating mental health and restoration of medical profession's advocacy and equity.

\section{Background}

Corona virus disease 2019 (COVID-19) pandemic has generated a widespread distress in the general population and particularly among the frontline physicians. The world health organization (WHO) has voiced grave concerns about the mounting emotional toll and deteriorating mental and physical 
wellbeing of physicians during the COVID-19 pandemic worldwide (1). Similarly, the National Health Service, UK has reported a staggering rise in the emotional burdens and levels of anxiety among the physicians, which may adversely affect their decision-making abilities and professional conduct and behaviours (2). These already overstretched, understaffed and under resourced healthcare systems have succumbed to the ever-increasing pressure and public expectation to continue working optimally during the pandemic (3).

The physicians' psychological health, professional and personal life has been challenged during the pandemic by extra duty hours, workload burden and the fear of being infected in health-care facilities (4). In addition to the challenges on physicians' mental and physical wellbeing, a constellation of factors including stress, anxiety, depression and burnout during the COVID-19 outbreak have contributed to the deterioration in psychological health (5). These include, but are not limited to, personal worries, uncertainty, lockdowns and closures, and reduced social contact (6). Concomitantly, the surge in demand for all sections of medical care and the re-allocation of scarce health care resources by minimizing the level of care for other patients have raised several ethical concerns of equity and social justice (7).

During the unprecedented crisis, the complex matrix of personal, professional, and societal expectations for physicians has substantially undermined their confidence and performance. The physicians are facing issues about professional accountability and moral obligations, mostly due to a lack of guidance and legislative controls which would have given them the ability to choose between alternatives (8). In this perspective, the literature has reported an escalating rise in various forms of psychological distress in physicians including moral injury which "results from actions, or the lack thereof, that violates someone's moral or ethical code" $(9,10)$. A range of experiences constitute moral injury such as a perception of guilt, shame, revulsion, and animosity.

The compounding impact of psychological disconnect, frustration, compromised decision-making, resource relocation and working under pressure is believed to have adversely affected the physicians' personal and professional wellbeing during the COVID-19 outbreak (11). There is a dire need for concerted efforts to mitigate the impact of modifiable risk factors which can potentially sabotage the physicians' wellbeing and their ability to cope with the unprecedented crisis (12). Unfortunately, though an existing body of literature has reported some evidence of issues related to the physicians' psychological distress and their personal and professional predicaments, there remains a paucity of understanding on this topic. This review investigates the impact of COVID-19 on the psychological, personal and professional performance of physicians with a view to secure a remedial pathway in the medical field.

\section{Methods}

\section{Methods}


The search design of our systematic scoping review was based on the following stages as described by the Arksey and O'Malley's framework (13).

- Defining the research questions based on the context of scoping review using a triad of participants, concept, and context

- Identifying relevant studies that matched the defined research questions and purpose

- Selecting studies using the pre-determined inclusion and exclusion criteria

- Charting the data

- Summarizing and reporting the results

Our search strategy did not apply the optional sixth requisite of Arksey and O'Malley's framework, the necessity of consultation with potential stakeholders, as this was not the main purpose of our research.

In the following sections, we have detailed each step of the Arksey and O'Malley's framework as used in our study.

\section{Defining research questions}

- How has COVID-19 affected the psychological health of physicians involved in the care of patients?

- What are the effects on the personal and professional wellbeing of the physicians?

\section{Identifying relevant studies: databases and process of data selection}

On June 17, 2021, the principal investigator (PI) (SSG) searched six databases: PubMed/Medline, Web of Science, Scopus, Science Direct, CINAHL and Psyclnfo. PubMed/Medline was the mainstay to systematically develop a search string, which was later extrapolated to other databases. We used ((Physicians) AND ((((Covid 19) OR (Sars CoV2)) OR (acute respiratory distress syndrome covid-19)) OR (2019-nCoV))) AND ((((moral injury) OR (moral distress)) OR (moral conflict)) OR (ethical dilemmas)) OR (psychological wellbeing) terms and text words for the English language original articles published between January 2020 and June 2021. Original article with quantitative, qualitative, and mixed methods study design were included. All selected keywords were searched using "abstract" and "article title" (alternatively "topic") and in the Medical Subject Headings (MeSH) terms or Thesaurus, where available. No filters or limitations were applied to retrieve the largest number of results. The articles published outside the defined time window, review and editorial articles, personal views and commentaries were excluded. Search for grey literature was conducted in the ProQuest Dissertation and Thesis. A full search log, including detailed search strings for all included information sources, results and notes are available in Appendix I.

\section{Selecting the studies: data extraction}


We used the Preferred Reporting Items for Systematic Reviews and Meta-analyses (PRISMA) guidelines for data mining and for the selection of studies for our scoping review (14). The three main constructs of our review included: concept (moral injury, moral distress, moral conflict, ethical dilemmas, medical professionalism), context (COVID-19) and participants (physicians involved in COVID-19 care) using the pre-determined inclusion and exclusion criteria. In our scoping review, we followed a structured protocol for the screening and selection of studies and then to review, chart and extract articles using the Covidence v1.0 data extraction template (15). Researchers PM and INL reviewed all titles and abstracts retrieved during the initial search and grouped relevant articles for possible inclusion. To ensure consistency and research quality, SYG reviewed all initially selected titles and abstracts. Any conflicts were resolved by FRD and SSG. Later, FRD and SSG reviewed full text of the selected articles. To mitigate research bias, the entire search process was finally reviewed by SSG, FRD and SYG. We resolved research disagreements and disputes through discussions until a consensus was reached. The final selection of the identified articles was considered for data extraction.

\section{Charting the data: data analysis}

Each article that qualified for the full-text review was independently reviewed by SSG and FRD. Covidence platform was used for the organization of the descriptive information (e.g., authors, year, type of article, study purpose, research design, participants, and ethical approval). A descriptive-analytical approach was applied for the charting and analysis of the key findings and recommendations emanating from the selected studies (16). FRD, SSG, INL and PM charted the articles separately. Later, all five authors conferred, a consensus was reached, and all overarching key themes were identified.

\section{Collating, summarizing, and reporting the results: data synthesis}

We performed a descriptive analysis (e.g., year of publication, country, health professionals' disciplines and study purposes) using Excel and performed a thematic analysis by following a grounded theory approach (17). The emerging themes and subthemes were further analyzed, discussed, and regrouped until a consensus was reached. Over the course of the analysis, the identified five themes were refined using the hierarchical clustering strategy (18), where we labelled each keyword as a descriptor, and then merged descriptors with maximum similarity into a subtheme. This similarity-based merger continued until all individual descriptors were collated into subthemes.

\section{Results}

Our initial search retrieved 875 titles, and after removing 272 duplicates and retaining only English language publications, we found 603 titles for further screening using the abstract analysis. This process further eliminated 518 titles which did not meet the inclusion criteria. A total of 83 full text articles were further reviewed for their eligibility. After full paper review, we included 28 articles in our scoping review for deeper analysis. The entire process using the PRISMA guidelines is illustrated in Figure 1. 


\section{[Insert Figure 1 here]}

According to the yearly publication pattern of the selected 28 articles, six articles were published in 2020 and 22 were published until June 17th, 2021. Three-quarters of the studies were multi-centre (75\%), and the majority of the studies were conducted in hospitals (22/79\%), while other sites included hospices, nursing homes and clinics. A graphical representation of the countries of origin of the selected 28 studies is displayed in Figure 2.

\section{[Insert Figure 2 here]}

Most studies (9/32\%) originated in the USA, while other studies were based in the Netherlands (3/11\%), United Kingdom, Canada, and France. Quantitative methodology was the most popular research design among the selected 28 studies, (16/57\%), where the researchers used cross-sectional surveys to acquire data as shown in Figure 3.

\section{[Insert Figure 3 here]}

Qualitative studies made up the next largest group (7/25\%) where semi-structured interviews, focus group discussions and one open-ended questionnaire were used. The remaining five studies, (5/18\%), used a mixed methods approach for the data collection. The average sample size of the 28 studies was 637, while a smaller number of participants were recruited in qualitative studies, ranging from 22 to 187 . A few of the quantitative studies had participants greater than 1000, i.e., 1050 (19), 1500 (20), 3006 (21), 4773 (22) and 1606 (23)). Its noteworthy that, out of all survey-based studies, only three studies had a response rate of $50 \%$ or greater $(3 / 19 \%,(24-26)$, while five did not explicitly report any response rate. Importantly, 27 (96\%) studies provided clear ethical statements either by institutional review board approval, exemption, or by stating that ethical approval was not necessary. A breakdown of the descriptive analysis of the physicians' medical subspecialties involved in the research from the selected 28 articles is shown in Table 1. 
Table 1

Descriptive analysis showing the breakdown of the physicians' medical subspecialties involved in the research from the selected articles $(n=28)$

\begin{tabular}{|ll|}
\hline Specialty of Practice & Number of studies \\
\hline Undeclared or diverse multispecialty* & 9 \\
\hline Surgical subspecialties & 6 \\
\hline Medical subspecialties & 11 \\
\hline Intensive medicine & 5 \\
\hline Emergency medicine & 10 \\
\hline Anaesthesia & 5 \\
\hline Psychiatry & 7 \\
\hline Paediatrics & 2 \\
\hline Obstetrics and gynaecology & 2 \\
\hline Family medicine & 2 \\
\hline Radiology & 1 \\
\hline
\end{tabular}

In terms of study populations, 10 of the 28 studies did not provide a breakdown of the gender groups. In the remaining 18, the total sample size was 15,509 and women made up almost two thirds of the participants $(9,827 / 63 \%)$. A total of seven studies had a primary research objective focused on exploring ethical dilemmas and subsequent decision-making skills of the participants. Other key research objectives of the selected studies in our scoping review are outlined in Table 2. 
Table 2

Leading research objectives of the selected studies about physicians' psychological health and personal and professional wellbeing in COVID-19 $(n=28)$

$\begin{array}{ll}\text { Objectives } & \begin{array}{l}\text { Number of } \\ \text { studies }\end{array}\end{array}$

Explore ethical dilemmas and subsequent decision-making skills 7

Establish impact on quality of patient care 5

Describe the personal experiences, concerns \& challenges while working during the pandemic

Assess psychological health parameters - burnout, mental health issues 7

Explore correlates of moral injury and distress 4

Identify the stressors that threaten physicians' wellbeing 5

Explore the support structures for promoting physician's emotional wellbeing 4

*Some research papers had more than one identifiable key objective.

Our iterative review process yielded five themes along with their relevant subthemes; mental health, individual challenges, decision-making, change in patient care, and support services Figure 4.

[Insert Figure 4 here]

Each theme along with its subthemes is elaborated in the following sections. A detailed tabular representation of themes can be found in Appendix II.

\section{Theme l: Mental health}

This theme was identified in (96\%) studies and, therefore, we ranked it as the first and the foremost challenge to physicians during the pandemic. The studies demonstrating an adverse impact of COVID-19 on the mental health of physicians included (19), (20), (21), (22), (23), (24), (25), (26), (27), (28), (29), (30), (29), (31), (32), (33), (34), (35), (36), (37), (38), (39), (40), (41), (42), (43), (44) and (45). The reviewed body of literature has reported that witnessing patients' sufferings unduly provoked sorrow, grief, and emotional distress among the frontline physicians. This phenomenon prevented them from working in the patient's best interest. Unsurprisingly, several physicians were found to suffer from mental exhaustion, burnout, sleeping disorders, worsening psychological wellbeing and compassion fatigue. Feelings of guilt, insecurity and fear triggered a sense of hopelessness, which led to depression particularly among the physicians dealing with COVID-19 deaths. Cynicism and detachment became typical coping strategies. Others felt lonely, isolated, and experienced a feeling of losing control, bringing about uncertainty which undermined their judgment and confidence.

\section{Theme II: Individual challenges}


This theme was developed from the referred studies; (19), (20), (21), (22), (24), (25), (26), (27), (28), (30), (31), (32), (34), (35), (36), (37), (38), (40), (39), (41), (42), (43), (44), and (45). There were two relevant subthemes linked to this theme: personal challenges and professional challenges. We found a plenty of evidence that during the pandemic, physicians were under tremendous pressure to maintain their personal and professional lives. They experienced sleep deprivation, physical exhaustion, and a decreased quality of life. The family lives of physicians were profoundly disturbed by them staying in hospitals or rental accommodation due to the fear of transmitting COVID-19 to family members. The physicians also faced serious professional challenges owing to feelings of deprivation, powerlessness in decision-making, shortage of personal protective equipment and limited access to testing. Scarce resources and inadequate infrastructure exacerbated the unfair work distribution as did the frequent changes in protocols bringing about both hierarchical and collegial rifts.

\section{Theme III: Decision-making}

The decision-making theme was located in the selected studies; (78.57\%), (19), (20), (22), (23), (24), (26), (28), (30), (29), (31), (32), (46), (33), (34), (35), (36), (37), (38), (40), (39) and (41). Our research generated three explicit subthemes of rationing care and triaging decisions, institutional or hierarchical impact on the individual's decision-making outcomes and skills and strategies to improve decision-making. In this research, we found an abundance of evidence that, during the COVID-19 outbreak, the physicians' decision-making became constrained. Triaging decisions and rationing the type of care were based on the patients' age, cognitive status, and the prognosis for survival. The immediacy of life and death decisions without further investigations and management breached ethical principles in the medical field (31). In contrast to the physicians' practice being mediated by the hospital's code of practice, the guidance from institutions became flawed and erratic. Consequently, the core patients' needs such as family visits and patient-doctor consultations were withdrawn, which provoked sentiments of futility and redundancy among physicians. There was some evidence that, in order to circumvent these challenges to decision-making, the workplace focused on shared multi-disciplinary activity, which contributed towards improved team cohesion under testing conditions.

\section{Theme IV: Change in patient care}

This theme was identified in the referred studies; (19), (20), (21), (22), (23), (24), (25), (26), (28), (30), (29), (31), (32), (46), (33), (34), (35), (36), (37), (38), (39), (42), (43) and (44). Three subthemes were identified under this theme: suboptimal care provision, doctor-patient interactions, and physicians' duty of care. The body of reviewed literature showed that the provision of healthcare during the outbreak was suboptimal. A paradigm shift in patient care was witnessed. The imposed restrictions were focused on minimizing infection spread, thus resulting in delayed and compromised care. Visitations to the loved ones, considered to be a vital part of the holistic recovery of patients, were reduced or suspended to prevent COVID-19 contamination. Likewise, there was a change in the doctor-patient interactions as they became depersonalized, primarily triggered by the concealment of facial expressions by mask wearing.

Furthermore, this concealment deprived the anxious and terminally sick patients of the essential non- 
verbal facial cues to comfort them (34). Ultimately, with intimacy lessened, the human bond between doctor and patient was lost.

The physicians' duty of care was adversely affected as their actions were impeded by new guidance codes laid down by hospital governance. Leading physicians had to think up creative ways to deliver care such as employing adjunct services to honor patients' wishes and experimenting different strategies that were not based on evidence.

\section{Theme V: Support services}

Finally, support services emerged as a main theme from studies; (20), (22), (23), (26), (28), (30), (31), (32), (34), (35), (36), (37), (38), (41) and (44). Two subthemes emanated from this main theme: near-peer support and institutional interventions. In this theme, a wide spectrum of diverse groups formed the nearpeer support structures they consisted of friends and family, colleagues, as well as other members of the hospital workforce and multi-professional teams. Although institutional support was in place, unfortunately, it was never sufficient to support the personal and professional wellbeing of physicians. Sporadically, some institutions offered those suffering from post-traumatic stress, anxiety, sleep disorders and stress-related issues one-to-one support. There was also a myriad of strategies that were hypothetically introduced such as flattened hierarchy, meditation, psychological counselling and rescheduling of the duty hours. However, all these coping strategies lacked commitment, rigor, and sustainability.

\section{Discussion}

This scoping review has reported a substantial impact of COVID-19 disease on the physicians' mental health, individual challenges, decision-making, and patient care. The reviewed body of literature has affirmed that the frontline physicians were subjected to significant stress due to the nature of their duties as they directly engaged with patients infected with COVID-19. The fear of disease transmission to their families, concerns about their own health and of loved ones, being stigmatized and forbidden, and working under extreme pressures have led to physicians' reporting emotional and physical burnouts. The scarcity of resources and redeployment of priorities by healthcare authorities created organizational dissonance and emotional distress and moral injury among the practicing physicians.

The fundamental message, stemming from our scoping review, is that the (re)allocation of resources and priorities have transformed the fiduciary nature of medical professionals' work towards a more utilitarian approach (47). Historically, medical professionals are recognized as moral agents with an inherent mission of justice and responsibility (48). This life-long commitment and accountability cannot be modified or suspended when resources become scarce as the core tenet of medical professionalism centres around the welfare and wellbeing of patients and society. The literature has shown that, during the pandemic, a great majority of physicians lost confidence and professional authority, primarily due to non-medical factors i.e., economic and political decisions (49) (50). 
Among the most reported changes in the physicians' mental health was moral distress which entails individuals' reactions to situations when they believe to know the right course of actions but they are unable to do it (51). During the COVID-19 crisis, physicians experienced moral distress when their personal and professional ethos conflicted with their institutional protocols or expectations. One of the adverse manifestations of moral distress is moral injury which was found to be prevalent among the frontline physicians dealing with the coronavirus infected patients (52). Moral injury contains a myriad of element 1) moral dissonance; 2) a sense of guilt, shame and existential conflicts; and 3) the presence of depression, anger, and anxiety (53). During the COVID-19 pandemic, physicians experienced moral injury as they committed unintentional errors leading to mortality or morbidity, due to their inability to prevent harm or death, the transgression of colleagues, supervisors or institutions who clashed with individuals' beliefs, or dealing with leaders who did not take full responsibility for the adverse clinical outcomes (54).

Wang et al., have reported that physicians faced substantially more fear, depression and psychological impairments compared to hospital administrators (55). This finding shed light on the varying degrees of stress and pressure sustained by different hierarchical strata in the same institution. In their survey-based study by Maftei and Holman, using the moral injury events scale, the investigators demonstrated comparably high levels of self-reported negative physical and emotional stress in the COVID-19 and the non-COVID-19 treating groups of physicians (53). The authors have argued that, regardless of the COVID19 or non-COVID-19 workforce assignment, medical professionals invariably faced similar workplace related stress, practice similar procedures, and followed medical instructions with a similar rigor and spirit. This review calls for urgent task-specific rehabilitation and remedial programs for the affected medical professionals catering for and supporting their personal and professional realms.

The reported uncertainty and powerlessness of physicians during the pandemic has led to subjective decision-making practices (56). The decision-making theme in our scoping review has thoroughly illustrated that priority settings dilemmas, evolving demands, and uncovered needs have forecasted uncertainty and disbelief among physicians (57). In an interesting study by Idilbi et al., the investigators sought physicians' preferences for the allocation of a ventilator to one of the three COVID-19 infected patients; an 80-year-old man without cognitive illness, a 50-year-old man with Alzheimer's disease (AD) or an 80-year-old man with $A D(58)$. A three-quarters of the respondents placed the 80-year-old man with $A D$ as the last choice, while they were equally divided about the selection of other patients. Similarly, a plethora of research has shown that the priorities of medical care have changed dramatically and key decisions were based on age, gender, ethnicity, life expectancy and associate comorbidities (59) (60). On the same note, rationing, triaging, redeployment of expertise, postponement of non-urgent cases, suboptimal substitutes and a lack of shared decision-making were prevalent in the reviewed literature. Neves et al., have coined this change in practice as 'how to decide who lives and who dies?' (61). From a different perspective, the postponement of elective cases in a wide range of medical and surgical specialties led to unforeseen complications (62).

In this scoping review, we have identified a paradigm change in the pattern of patient care during the COVID-19 pandemic. As reported by Dewar et al., some physicians did not notice any change in their 
practice, while a vast number of intensivists, ethicists, and general practitioners felt 'an entirely new experience' in their clinical work (63). To combat the crisis, some physicians created alternative management pathways by adopting unorthodox and non-standardized substitutes, which could be harmful and suboptimal (64). Some researchers have argued that, in a desperate attempt to do everything to save lives, sometimes physicians experimented with aggressive treatment options and delayed end-of-life decisions which added distress and frustration among families and society $(65,66)$. The changing optics of the physicians' approach towards equity and justice especially in the critical care units cannot be overemphasized. Arabi et al., highlighted the pressing need for transformative changes in critical care units by enhancing the ICU bed capacity, redesigning the units for COVID-19 infected patients, flexible timetabling for staff, sustainable supply chains for equipment, and the development of ICU triage protocols (67).

Its noteworthy, that several physicians reported that they were not formally engaged in the institutional planning and implementation phases of new policies during the pandemic (68). Thereupon, the physicians developed their own personal policies to resolve ambiguities and uncertainties in patient care and utterly disregarded the institutional protocols (69). Thus creating major inconsistencies in the workflow leading to heterogeneous responses and outcomes among institutions. Additionally, physicians were suspicious about changing triage protocols using ambiguous or limited resources. Finally, physicians unfortunately experienced contentious conversations with patients and their families who thought that the physicians' expertise and support services were far below the proclaimed standards of care (70).

\section{Recommendations}

We have summarized the recommendations by appraising the key findings from the literature which can be essentially pitched at micro (personal), meso (institutional) and macro (society) levels (Figure 5).

\section{[Insert Figure 5 here]}

At the micro level, near-peer support, meditation and mindfulness programs can enhance physicians' psychological health. Similarly, at meso level we identified the need to pay maximum attention to institutional support by creating allocation algorithms for resources, capacity, taskforce, and budgeting decisions. Likewise, group deliberations among institutional leaders and physicians to address ethical issues and operational tasks are essential. Formal involvement of physicians in institutional planning, establishing contingency plans, structured rehabilitation programs and clear policies to mitigate moral distress for physicians and support staff. Lastly at the macro level, physicians' contributions by public should be recognized and rewarded.

\section{Conclusion}

This scoping review highlights the rise in mental distress, moral injury, and a wide range of psychopathological events among practicing physicians during the COVID-19 pandemic. There was a 
paradigm change in the quality of patient care. A myriad of factors including workload, stress, uncertainty, lack of morality, absence of professional advocacy and authority, and dismal institutional services contributed to the deteriorating wellbeing of physicians. There was a perceived loss of professional and legislative controls thus undermining professional advocacy and equity. This research calls for the identification and resurrection of poor workplace conditions, mental and physical rehabilitation of the affected physicians, development of plans to build individual resilience, and practice shared decision-making. Lastly, medical professionals should be allowed to serve the ailing humanity independently without any economic or political influence.

\section{Declarations}

\section{Ethics approval and consent to participate}

Not applicable

\section{Consent for publication}

Not applicable

\section{Availability of data and materials}

All data generated or analysed during this study are included in this published article [and its supplementary information files].

\section{Competing interests}

The authors declare that they have no competing interests.

\section{Funding}

This research is funded by The School of Postgraduate Studies and Research of The Royal College of Surgeons Ireland - Medical University Bahrain.

\section{Authors' contributions}

All authors (SSG, PM, INL, SYG and FRD) contributed substantially to conceiving the idea. SSG searched the databases and developed the search strategy. All authors later validated and approved the search strategy. PM and INL reviewed all titles and abstracts retrieved during the initial search and grouped relevant articles for possible inclusion. To ensure consistency and research quality, SYG reviewed all initially selected titles and abstracts. Any conflicts were resolved by FRD and SSG. Later, working in two groups PM and INL, FRD and SSG independently reviewed full text of the selected articles, extracted, analysed and interpreted the data and came up with the thematic analysis. To mitigate research bias, the entire search process was finally reviewed by SSG, FRD and SYG. Later SYG individually evaluated the 
search strategy, tweaked the data mining process, revised and improved the intellectual content of the initial draft. All members agreed to take responsibility for the final draft.

\section{Acknowledgements}

Not applicable

\section{References}

1. Wu M, Han H, Lin T, Chen M, Wu J, Du X, et al. Prevalence and risk factors of mental distress in China during the outbreak of COVID-19: a national cross-sectional survey. Brain and behavior. 2020;10(11):e01818.

2. Cipolotti L, Chan E, Murphy P, van Harskamp N, Foley JA. Factors contributing to the distress, concerns, and needs of UK Neuroscience health care workers during the COVID-19 pandemic. Psychology and Psychotherapy: Theory, Research and Practice. 2021;94:536-43.

3. Guraya SY. Transforming laparoendoscopic surgical protocols during the COVID-19 pandemic; big data analytics, resource allocation and operational considerations. International Journal of Surgery. 2020;80:21-5.

4. Mosheva M, Hertz-Palmor N, Dorman Ilan S, Matalon N, Pessach IM, Afek A, et al. Anxiety, pandemicrelated stress and resilience among physicians during the COVID-19 pandemic. Depression and anxiety. 2020;37(10):965-71.

5. Viertiö S, Kiviruusu O, Piirtola M, Kaprio J, Korhonen T, Marttunen M, et al. Factors contributing to psychological distress in the working population, with a special reference to gender difference. BMC public health. 2021;21(1):1-17.

6. Shechter A, Diaz F, Moise N, Anstey DE, Ye S, Agarwal S, et al. Psychological distress, coping behaviors, and preferences for support among New York healthcare workers during the COVID-19 pandemic. General hospital psychiatry. 2020;66:1-8.

7. Ćurković $M$, Košec $A$, Ćurković D. Medical professionalism in times of COVID-19 pandemic: is economic logic trumping medical ethics? Internal and Emergency Medicine. 2020;15(8):1585-6.

8. Arora A, Arora A. Ethics in the age of COVID-19. Internal and emergency medicine. 2020;15:889-90.

9. Greenberg N, Docherty M, Gnanapragasam S, Wessely S. Managing mental health challenges faced by healthcare workers during covid-19 pandemic. bmj. 2020;368.

10. Dean W, Jacobs B, Manfredi RA. Moral injury: The invisible epidemic in COVID health care workers. Annals of Emergency Medicine. 2020;76(4):385-6.

11. Roca J, Canet-Vélez O, Cemeli T, Lavedán A, Masot O, Botigué T. Experiences, emotional responses, and coping skills of nursing students as auxiliary health workers during the peak COVID-19 pandemic: A qualitative study. International Journal of Mental Health Nursing. 2021.

12. Elhadi M, Msherghi A, Elgzairi M, Alhashimi A, Bouhuwaish A, Biala $M$, et al. The mental well-being of frontline physicians working in civil wars under coronavirus disease 2019 pandemic conditions. 
Frontiers in Psychiatry. 2020;11.

13. Daudt HM, van Mossel C, Scott SJ. Enhancing the scoping study methodology: a large, interprofessional team's experience with Arksey and O'Malley's framework. BMC medical research methodology. 2013;13(1):1-9.

14. Guraya SY, Abdalla ME. Determining the effectiveness of peer-assisted learning in medical education: a systematic review and meta-analysis. Journal of Taibah University Medical Sciences. 2020;15(3):177-84.

15. Babineau J. Product review: covidence (systematic review software). Journal of the Canadian Health Libraries Association/Journal de l'Association des bibliothèques de la santé du Canada. 2014;35(2):68-71.

16. Maghbouli N, Mafinejad MK, Pourhassan S. Relationship Between Mentor Retention and the Role of Personality: A Descriptive-Analytical Study. Journal of Multidisciplinary Healthcare. 2020;13:549.

17. Watling CJ, Lingard L. Grounded theory in medical education research: AMEE Guide No. 70. Medical teacher. 2012;34(10):850-61.

18. Hourdakis N, Argyriou M, Petrakis EG, Milios EE. Hierarchical Clustering in Medical Document Collections: the BIC-Means Method. J Digit Inf Manag. 2010;8(2):71-7.

19. Mion G, Hamann P, Saleten M, Plaud B, Baillard C. Psychological impact of the COVID-19 pandemic and burnout severity in French residents: A national study. The European Journal of Psychiatry. 2021;35(3):173-80.

20. Mellins CA, Mayer LES, Glasofer DR, Devlin MJ, Albano AM, Nash SS, et al. Supporting the well-being of health care providers during the COVID-19 pandemic: The CopeColumbia response. Gen Hosp Psychiatry. 2020;67:62-9.

21. Wang Z, Harold KG, Tong Y, Wen J, Sui M, Liu H, et al. Moral injury in Chinese health professionals during the COVID-19 pandemic. Psychol Trauma. 2021.

22. Juvet TM, Corbaz-Kurth S, Roos P, Benzakour L, Cereghetti S, Moullec G, et al. Adapting to the unexpected: Problematic work situations and resilience strategies in healthcare institutions during the COVID-19 pandemic's first wave. Safety Science. 2021;139.

23. Miljeteig I, Forthun I, Hufthammer KO, Engelund IE, Schanche E, Schaufel M, et al. Priority-setting dilemmas, moral distress and support experienced by nurses and physicians in the early phase of the COVID-19 pandemic in Norway. Nurs Ethics. 2021;28(1):66-81.

24. Caruana EJ, Patel A, Kendall S, Rathinam S. Impact of coronavirus 2019 (COVID-19) on training and well-being in subspecialty surgery: A national survey of cardiothoracic trainees in the United Kingdom. J Thorac Cardiovasc Surg. 2020;160(4):980-7.

25. Abdessater M, Roupret M, Misrai V, Matillon X, Gondran-Tellier B, Freton L, et al. CoVID19 pandemic impacts on anxiety of French urologist in training: Outcomes from a national survey. Prog Urol. 2020;30(8-9):448-55.

26. O'Neal L, Heisler M, Mishori R, Haar RJ. Protecting providers and patients: results of an Internet survey of health care workers' risk perceptions and ethical concerns during the COVID-19 pandemic. 
Int J Emerg Med. 2021;14(1):18.

27. Maftei A, Holman AC. The prevalence of exposure to potentially morally injurious events among physicians during the COVID-19 pandemic. Eur J Psychotraumatol. 2021;12(1):1898791.

28. Rao H, Mancini D, Tong A, Khan H, Santacruz Gutierrez B, Mundo W, et al. Frontline interdisciplinary clinician perspectives on caring for patients with COVID-19: a qualitative study. BMJ Open. 2021;11(5):e048712.

29. Idilbi N, AboJabel H, Werner P. Examining hospital staff members' preferences for allocating a ventilator to a COVID-19 patient with and without Alzheimer's disease. Geriatr Nurs. 2021;42(4):78791.

30. Sukhera J, Kulkarni C, Taylor T. Structural distress: experiences of moral distress related to structural stigma during the COVID-19 pandemic. Perspect Med Educ. 2021:1-8.

31. Kana L, Shuman A, De Vries R, Firn J. Taking the burden off: a study of the quality of ethics consultation in the time of COVID-19. J Med Ethics. 2021.

32. Kreh A, Brancaleoni R, Magalini SC, Chieffo DPR, Flad B, Ellebrecht N, et al. Ethical and psychosocial considerations for hospital personnel in the Covid-19 crisis: Moral injury and resilience. PLoS One. 2021;16(4):e0249609.

33. Sizoo EM, Monnier AA, Bloemen M, Hertogh C, Smalbrugge M. Dilemmas With Restrictive Visiting Policies in Dutch Nursing Homes During the COVID-19 Pandemic: A Qualitative Analysis of an OpenEnded Questionnaire With Elderly Care Physicians. J Am Med Dir Assoc. 2020;21(12):1774-81 e2.

34. Viswanathan R, Myers MF, Fanous AH. Support Groups and Individual Mental Health Care via Video Conferencing for Frontline Clinicians During the COVID-19 Pandemic. Psychosomatics. 2020;61(5):538-43.

35. Butler CR, Wong SPY, Wightman AG, O'Hare AM. US Clinicians' Experiences and Perspectives on Resource Limitation and Patient Care During the COVID-19 Pandemic. JAMA Netw Open. 2020;3(11):e2027315.

36. Eftekhar Ardebili M, Naserbakht M, Bernstein C, Alazmani-Noodeh F, Hakimi H, Ranjbar H. Healthcare providers experience of working during the COVID-19 pandemic: A qualitative study. Am J Infect Control. 2021;49(5):547-54.

37. Gaucher N, Trottier ED, Cote AJ, Ali H, Lavoie B, Bourque CJ, et al. A survey of Canadian emergency physicians' experiences and perspectives during the COVID-19 pandemic. CJEM. 2021;23(4):466-74.

38. Huffman EM, Athanasiadis DI, Anton NE, Haskett LA, Doster DL, Stefanidis D, et al. How resilient is your team? Exploring healthcare providers' well-being during the COVID-19 pandemic. Am J Surg. 2021;221(2):277-84.

39. McNeil MJ, Kaye EC, Vedaraju Y, Baker JN, Devidas M, Downing J, et al. Global Experiences of Pediatric Palliative Care Teams During the First 6 Months of the SARS-CoV-2 Pandemic. J Pain Symptom Manage. 2021.

40. Korkmaz S, Kazgan A, Cekic S, Tartar AS, Balci HN, Atmaca M. The anxiety levels, quality of sleep and life and problem-solving skills in healthcare workers employed in COVID-19 services. J Clin 
Neurosci. 2020;80:131-6.

41. Shalhub S, Mouawad NJ, Malgor RD, Johnson AP, Wohlauer MV, Coogan SM, et al. Global vascular surgeons' experience, stressors, and coping during the coronavirus disease 2019 pandemic. J Vasc Surg. 2021;73(3):762-71 e4.

42. Doherty AM, Colleran GC, Durcan L, Irvine AD, Barrett E. A pilot study of burnout and long covid in senior specialist doctors. Ir J Med Sci. 2021:1-5.

43. Hesselink G, Straten L, Gallée L, Brants A, Holkenborg J, Barten DG, et al. Holding the frontline: a cross-sectional survey of emergency department staff well-being and psychological distress in the course of the COVID-19 outbreak. BMC Health Serv Res. 2021;21(1):525.

44. Ezzat A, Li Y, Holt J, Komorowski M. The global mental health burden of COVID-19 on critical care staff. Br J Nurs. 2021;30(11):634-42.

45. Maraqa B, Nazzal Z, Zink T. Mixed Method Study to Explore Ethical Dilemmas and Health Care Workers' Willingness to Work Amid COVID-19 Pandemic in Palestine. Front Med (Lausanne). 2020;7:576820.

46. Onwuteaka-Philipsen BD, Pasman HRW, Korfage IJ, Witkamp E, Zee M, van Lent LG, et al. Dying in times of the coronavirus: An online survey among healthcare professionals about end-of-life care for patients dying with and without COVID-19 (the CO-LIVE study). Palliat Med. 2021;35(5):830-42.

47. Lai J, Ma S, Wang Y, Cai Z, Hu J, Wei N, et al. Factors associated with mental health outcomes among health care workers exposed to coronavirus disease 2019. JAMA network open. 2020;3(3):e203976-e.

48. López L, Dyck AJ. Educating physicians for moral excellence in the twenty-first century. Journal of Religious Ethics. 2009;37(4):651-68.

49. Mion G, Hamann P, Saleten M, Plaud B, Baillard C. Psychological impact of the COVID-19 pandemic and burnout severity in French residents: A national study. The European Journal of Psychiatry. 2021.

50. Robert R, Kentish-Barnes N, Boyer A, Laurent A, Azoulay E, Reignier J. Ethical dilemmas due to the Covid-19 pandemic. Annals of intensive care. 2020;10(1):1-9.

51. Doka K, Rushton CH, Thorstenson TA. Healthcare ethics forum'94: caregiver distress: if it is so ethical, why does it feel so bad? AACN Advanced Critical Care. 1994;5(3):346-52.

52. Zhizhong W, Koenig HG, Yan T, Jing W, Mu S, Hongyu L, et al. Psychometric properties of the moral injury symptom scale among Chinese health professionals during the COVID-19 pandemic. BMC psychiatry. 2020;20(1):1-10.

53. Maftei A, Holman A-C. The prevalence of exposure to potentially morally injurious events among physicians during the COVID-19 pandemic. European Journal of Psychotraumatology. 2021;12(1):1898791.

54. Williamson V, Murphy D, Greenberg N. COVID-19 and experiences of moral injury in front-line key workers. Oxford University Press UK; 2020. 
55. Lu W, Wang H, Lin Y, Li L. Psychological status of medical workforce during the COVID-19 pandemic: A cross-sectional study. Psychiatry research. 2020;288:112936.

56. Rajan D, Koch K, Rohrer K, Bajnoczki C, Socha A, Voss M, et al. Governance of the Covid-19 response: a call for more inclusive and transparent decision-making. BMJ Global Health. 2020;5(5):e002655.

57. Miljeteig I, Forthun I, Hufthammer KO, Engelund IE, Schanche E, Schaufel M, et al. Priority-setting dilemmas, moral distress and support experienced by nurses and physicians in the early phase of the COVID-19 pandemic in Norway. Nursing ethics. 2021;28(1):66-81.

58. Brooke J, Jackson D. Older people and COVID-19 isolation, risk and ageism. Journal of clinical nursing. 2020.

59. Mehta B, Jannat-Khah D, Fontana MA, Moezinia CJ, Mancuso CA, Bass AR, et al. Impact of COVID-19 on vulnerable patients with rheumatic disease: results of a worldwide survey. Rmd Open. 2020;6(3):e001378.

60. Razai MS, Kankam HK, Majeed A, Esmail A, Williams DR. Mitigating ethnic disparities in covid-19 and beyond. bmj. 2021;372.

61. Neves NM, Bitencourt FB, Bitencourt AG. Ethical dilemmas in COVID-19 times: how to decide who lives and who dies? Revista da Associação Médica Brasileira. 2020;66:106-11.

62. Guraya S. Combating the COVID-19 outbreak with a technology-driven e-flipped classroom model of educational transformation. Journal of Taibah University Medical Sciences. 2020;15(4):253.

63. Dewar B, Anderson JE, Kwok ES, Ramsay T, Dowlatshahi D, Fahed R, et al. Physician preparedness for resource allocation decisions under pandemic conditions: A cross-sectional survey of Canadian physicians, April 2020. PloS one. 2020;15(10):e0238842.

64. Abbasi J. "Abandoned" nursing homes continue to face critical supply and staff shortages as COVID19 toll has mounted. Jama. 2020;324(2):123-5.

65. Kip KE, Snyder G, Yealy DM, Mellors JW, Minnier T, Donahoe MP, et al. Temporal changes in clinical practice with COVID-19 hospitalized patients: Potential explanations for better in-hospital outcomes. medRxiv. 2020.

66. Ravdin PM. The lack, need, and opportunities for decision-making and informational tools to educate primary-care physicians and women about breast cancer chemoprevention. Cancer prevention research. 2010;3(6):686-8.

67. Arabi YM, Murthy S, Webb S. COVID-19: a novel coronavirus and a novel challenge for critical care. Intensive care medicine. 2020;46(5):833-6.

68. Butler CR, Wong SP, Wightman AG, O'Hare AM. US clinicians' experiences and perspectives on resource limitation and patient care during the COVID-19 pandemic. JAMA network open. 2020;3(11):e2027315-e.

69. Håkansson A, Fernández-Aranda F, Menchón JM, Potenza MN, Jiménez-Murcia S. Gambling during the COVID-19 crisis-a cause for concern. Journal of addiction medicine. 2020;14(4):e10. 
70. Vaillant GE, Sobowale NC, McARTHUR C. Some psychologic vulnerabilities of physicians. New England Journal of Medicine. 1972;287(8):372-5.

\section{Figures}
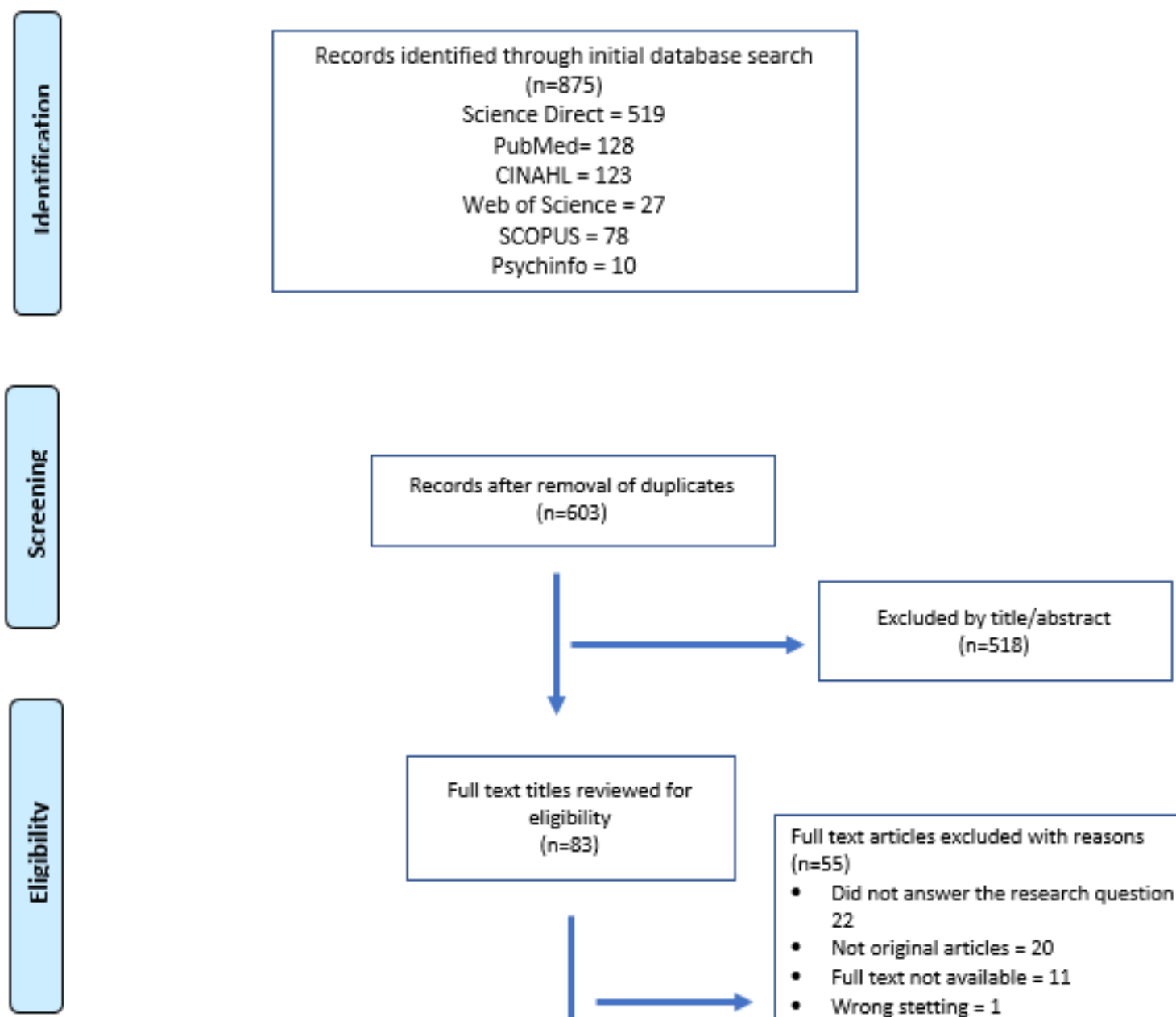

Full text titles reviewed for eligibility ( $n=83$ )

Full text articles excluded with reasons $(n=55)$

- Did not answer the research question = 22

- Not original articles $=20$

- Full text not available $=11$

- Wrong stetting = 1

- Not in English language = 1

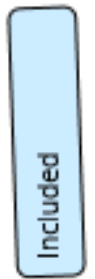

Final studies included for qualitative synthesis $(n=28)$

\section{Figure 1}

A flow chart for the selection of the studies about physicians' psychological health and personal and professional wellbeing in COVID-19 using the Preferred Reporting Items for Systematic Reviews and Meta-analyses (PRISMA) guidelines. 


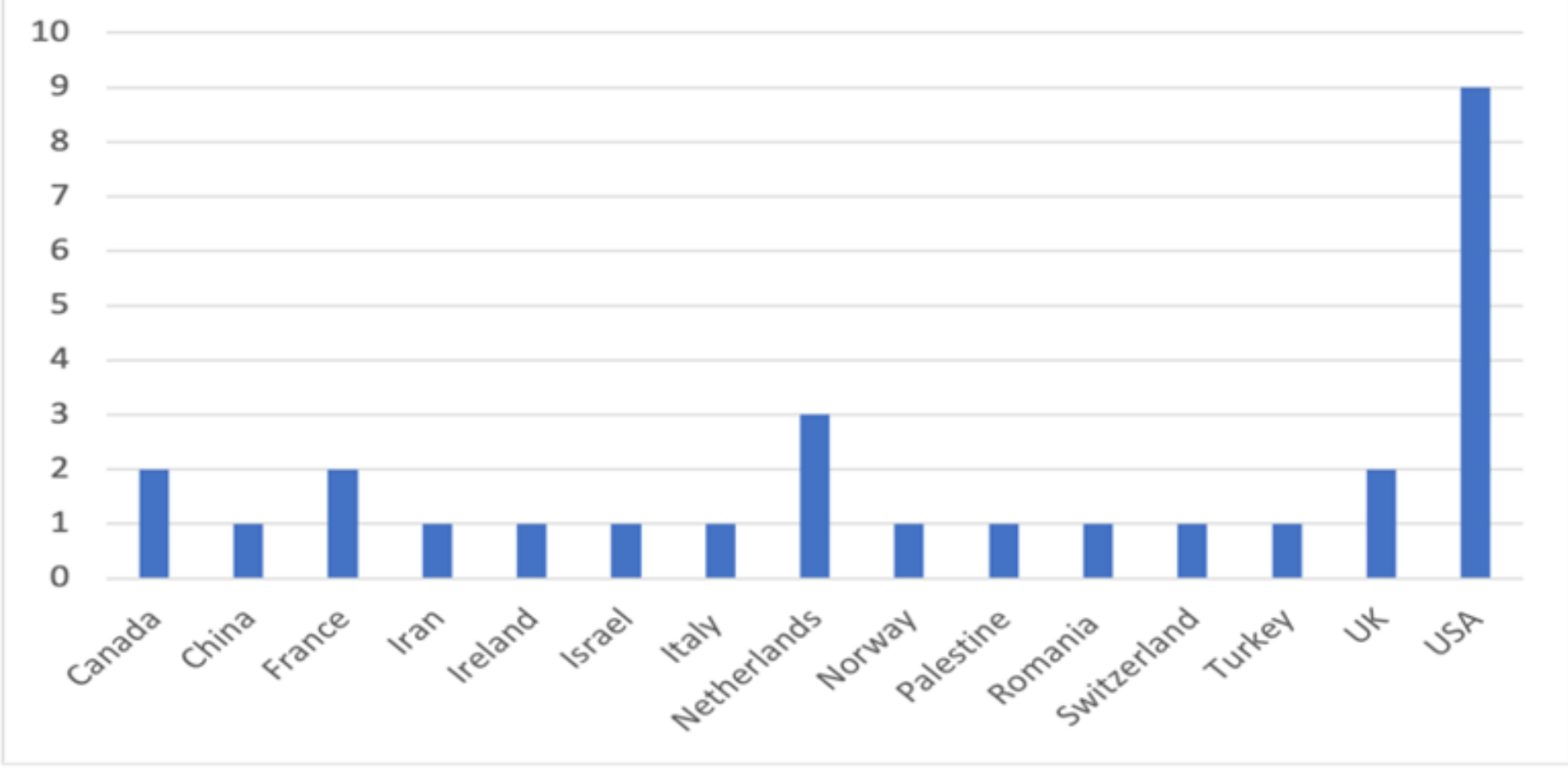

Figure 2

Country-wise representation of the selected studies about physicians' psychological health and personal and professional wellbeing in COVID-19 $(n=28)$

\section{Quanitative $(n=16)$}

- Cross-sectional surveys

\section{Qualitative $(n=7)$}

- Semi-structured interviews $(n=5)$

- Focus group discussion $(n=1)$

- Open-ended questionnaire $(n=1$

\section{Mixed methods $(n=5)$}

- Surveys, interviews \& focus groups $(n=1)$

- Survey and semistructured interviews $(n=1)$

- Survey and peer supervision discussions $(n=1)$

- Online questionnaires with subsets of openended questions $(n=1)$

- MCQ's \& free-text questions $(n=1)$ 
The range of research designs for the collection data in the selected studies about physicians' psychological health and personal and professional wellbeing in COVID-19 $(n=28)$

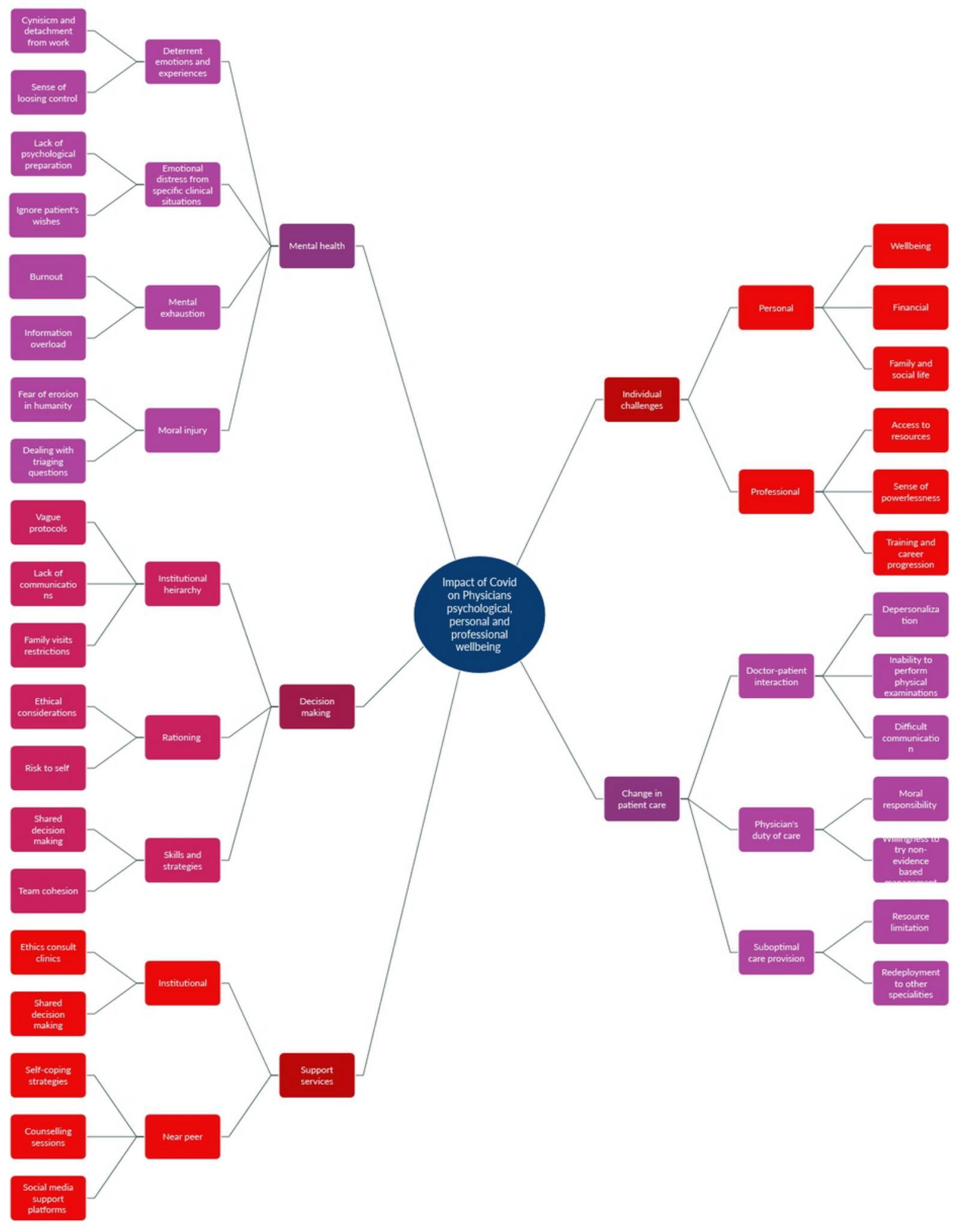

Figure 4 
Thematic analysis using a grounded theory approach.

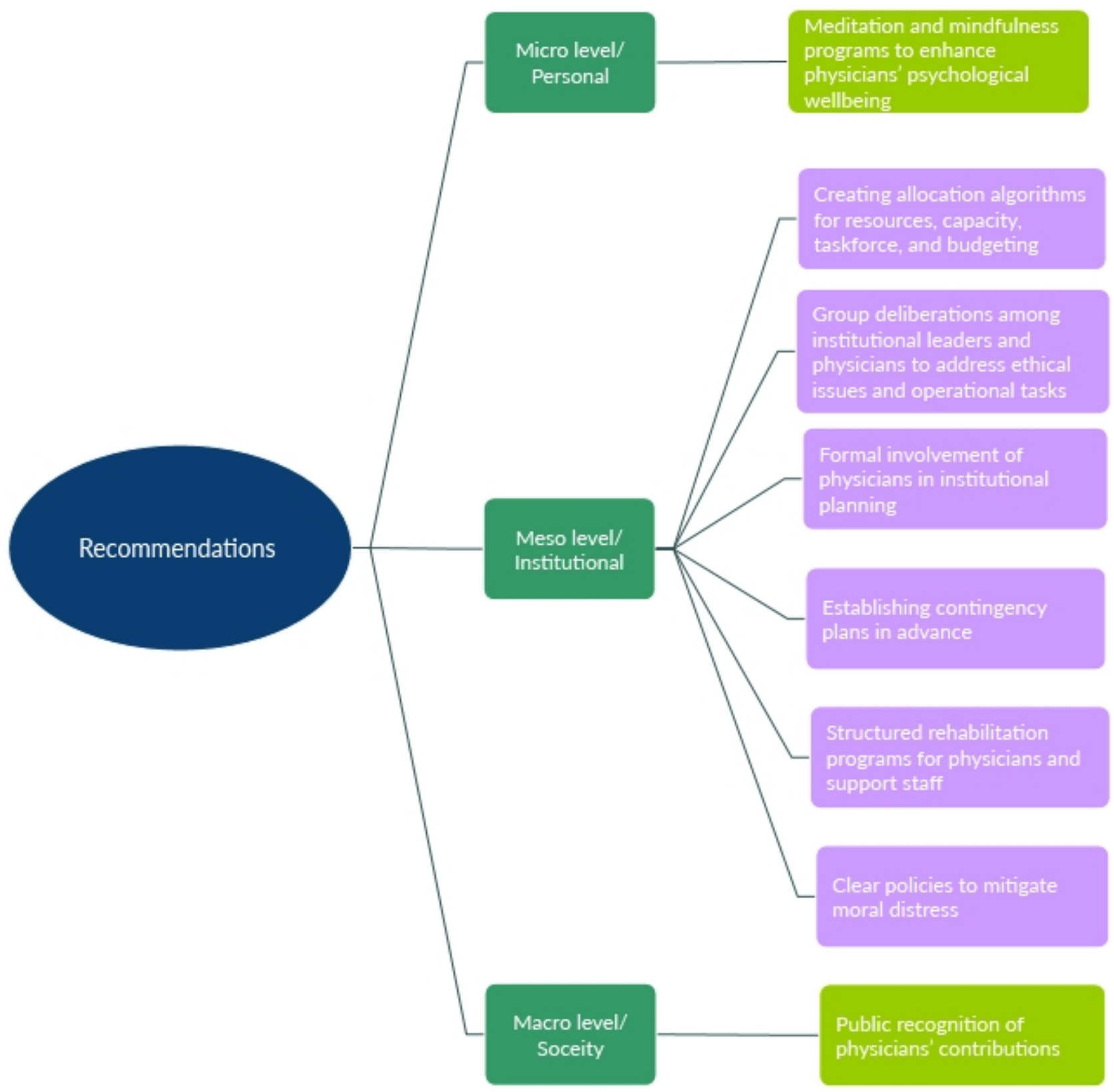

Figure 5

Recommendations for micro, meso and macrolevels to improve physicians psychological, physical and professional wellbeing.

\section{Supplementary Files}

This is a list of supplementary files associated with this preprint. Click to download.

- Appendixl.docx

- Appendixll.docx 